\title{
METHODS FOR DETERMINING WORST-SERVED CUSTOMERS
}

\author{
Tibor TERSZTYANSZKY \\ Hungarian Energy Office - Hungary \\ tersztyanszkyt@eh.gov.hu
}

\begin{abstract}
The paper outlines the service quality measurements in general and analyzes them from the customer's point of view. Applied procedures of worst-served customers including so-called guaranteed standards are introduced. From the overview the paper finds out that there are several but different methods for determining worstserved customers. The paper concludes that the definitions do not cover important fields from the customer's point of view e.g. voltage quality. The paper proposes to set stricter targets than Guaranteed standards for worst-served customers and gives comments on guaranteed standards if they are used for worst-served customers.
\end{abstract}

\section{INTRODUCTION}

The level of quality provided to the end user of electricity is determined by a variety of factors in different segments of the electricity supply chain. This paper focuses only on aspects influencing quality related to distribution and supply. The majority of consumers are well served; i.e. they have no or little problems with supply. Indices of service quality are invaluable in measuring system performance of supply quality. The average number and duration of interruptions per customer do not tell us anything about the customers who suffered the most outages, the bad quality of voltage, etc. Greater emphasis needs to be placed on the issues faced by worst served consumers.

\section{OUTLINE OF THE SERVICE QUALITY MEASURING}

There have been many different approaches to classifying the qualitative aspects of electricity supply, complicated further by the current practice of separating the functions of generation, supply, network operation, etc. Quality of service can be divided in commercial quality and power quality, and power quality can be differentiated as Continuity of the supply and Voltage quality. According to the international trends measuring the quality of service provided by electric companies are in the following areas [1,2]:

- Continuity of supply

- Commercial quality

- Voltage quality
In the following, we shall outline development of these fields from the customers' point of view.

\section{Continuity of supply}

System approach focuses on overall continuity through the measurement of average performance. The majority of indices in use provide a measure for the average number and duration of interruptions that took place or for the average time during which the supply was not available.

The average indices are invaluable in measuring system performance. They work well in benchmarking with other utilities, countries and are also useful for internal comparisons. They are vital in tracking outage trends and system reliability goals. Average standards and yearly rate of improvement standards are more appropriate for promoting overall improvement or maintaining quality.

From the customer's point of view the disadvantage of system indices is that they only provide information for the average customer, not for any individual customer. An individual customer is in principle only interested in the interruptions that impact its point of connection. Customer experiences don't necessarily correlate to system indices, and averages can hide a multitude of sins. For example, a $20 \%$ improvement in SAIFI may go unnoticed by the customer base. Conversely, a minute increase in SAIDI may indicate a large number of dissatisfied customers [3]. Suitable indicators for individual customers are the number and duration of interruptions experienced by the individual customers during a given year in the form of individual customer standards.

\section{Commercial Quality}

Commercial quality deals with the quality of the relationship from the non-technical point of view before the beginning or during the supply. It is important to potential customers in selecting a supplier and it takes effect at the moment of prospective customer requests for information or request to be connected to the network. Commercial quality covers many aspect of the relationship, although not all of services can be measured and regulated through standards or other instruments.

The most frequently used areas of commercial quality are as follows [2]:

- Connection of the customers (e.g. Cost estimation for connection) 
- Customer care (e.g. Punctuality of appointments with customers)

- Technical service (e.g. Solving problems related with voltage or meter)

- Meter reading and billing

Owing to the liberalization a distinction must be made between services provided by a supplier and those provided by a distributor.

There are mainly two types of quality standards for commercial quality: Guaranteed Standards and Overall Standards.

From the customer's point of view the Guaranteed Standards (GS) are more valuable as this system realizes a more personal perception of breaching the standards. GS refers to the service level which is expected to be fulfilled by the service provider. If the level is not met the company compensates the customer affected. In the case of Overall Standards (e.g. the minimum percentage of transactions during a predetermined time) no compensation is paid to customers in case of not meeting the standard. In some case the regulator can fine the company for breaching the standard under the so-called Other Available Requirements.

\section{Voltage quality}

The usefulness of electricity when there is no interruption is referred to as the voltage quality. Voltage quality can simply be described by deviations from nominal values for the voltage frequency and the voltage magnitude and by the distortion of the voltage wave shape. The voltage quality is expressed with respect to stated criteria. In order to decide whether the quality is good or poor, it is necessary to establish criteria to evaluate against. In Europe the most important norm as regards voltage characteristics of electricity supplied by public distribution networks is the CENELEC norm EN 50160. In general, this standard is applied but in some countries there are stricter requirements than EN 50160.

From the customer's point of view the main characteristics of the voltage has been becoming an important issue; which is due to inter alia an increase in the sensitivity of end user equipment over the past twenty to thirty years. When the voltage quality is very poor; several problems may arise when using electrical appliances and electrical processes; e.g. damage, flicker from the light.

\section{METHODS USED FOR DEFINITION OF WORST-SERVED CUSTOMERS}

Standardized definition of worst-served consumes. The standardized indicators for measuring the system average service quality are well elaborated on international and national level [1,4,5] but indicators for worst-served customers are missing. The IEEE Standard [4] - besides definition of system indicators - contains two indicators which can give information on worstserved customers:

$\checkmark$ "Customers Experiencing Multiple Interruptions" (CEMIn). This index indicates the ratio of individual customers experiencing more than a certain number (n) of interruptions to the total number of consumers served during a given period.

Note: This index is often used as a calculation within an incremented form

$\checkmark$ "Customers Experiencing multiple sustained interruption and momentary interruption events" (CEMSMIn). This index is the ratio of individual customers experiencing more than a certain number (n) of both sustained and momentary interruptions events to the total number of consumers served during a given period.

These indicators give information on a certain group of worst-served customers but they are related to an individual customer. We can state that a standardized definition of worst-served customers is missing.

Definition by Guaranteed standards. Service quality regulation focuses on the average level of quality but regulators are also concerned with protecting the worstserved customers. The instrument of minimum quality standards is a possibility to protect the customers in form of guaranteed standards. According to these standards each customer should be provided with at least a minimum level of guaranteed quality standards and determine the individual compensations to the affected customers where the company fails to meet the standards. From this point of view Guaranteed standards are called protecting "worst-served consumers" and presents both commercial quality and continuity of supply guaranteed standards e.g. guaranteed standards on long interruptions, making and keeping appointment [2,8].

\section{Definition related to individual customer standards.}

To protect the customers a number of European regulators set and maintain "individual customer standards" often in the form of guaranteed standards [1]. Single-customer continuity standards are generally expressed as the maximum yearly number of long (and short) interruption and/or a maximum duration or maximum yearly duration of interruptions, but can also concern other continuity indicators.

Single-customer continuity standards are thresholds applied to continuity indicators that have to be respected for every single customer connected to the network. The comparison shows large differences among the thresholds, ranging from 2 to 8 long interruptions per year for customers in urban contexts, and from 5 to more than 20 long interruptions per year for customers in rural contexts. The large differences among EU countries can be partly explained by differences in different regulatory approaches in setting standards [1]. A possible approach for regulators setting multiple interruption standards 
could involve looking at a given percentage of worstserved customers: In Italy, for instance, multiple interruption standards have been set in consideration of customers above the $90^{\text {th }}$ percentile in terms of number of long unplanned interruptions during the year [8]. In Hungary the percentage of consumers without electricity is monitored (Fig.1).

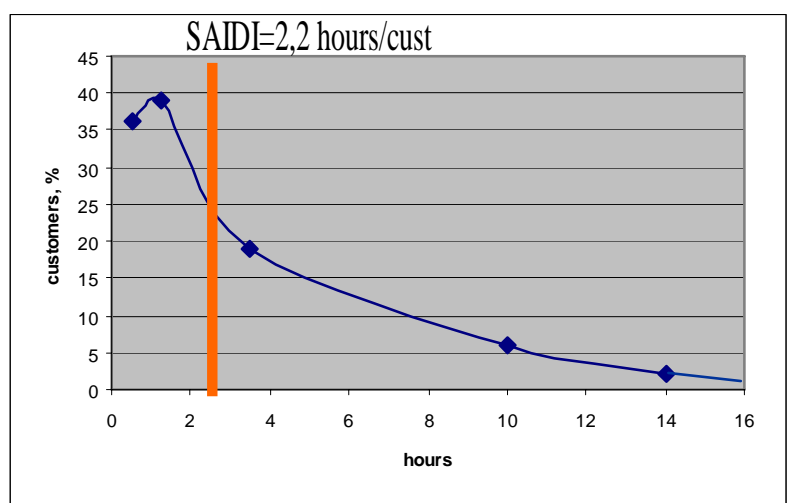

Fig. 1 percentage of consumers without electricity in Hungary in 2007

Definition related to feeders. Some regulators and network operators (outside of Europe but not exclusively) collect indices per feeder, where the values for the worstperforming feeders are reported, published and regulated. In the interest of this there are definitions [6]:

- "Feeder Average Interruption Frequency Index" (FAIFI) is the average number of service interruptions per customer served by the feeder.

- "Feeder Average Interruption Duration Index" (FAIDI) is the average number of service interruption minutes per customer served by the feeder.

The utility reporting system sometimes includes a more detailed account of outages for a calendar year with each index calculated for the utility's complete system, then broken down into individual circuits and analyzed to determine the contributing factors for the 10 worst performing circuits. [7].

Definition related to areas. Sometimes national regulators introduce special provisions targeting the worst performing areas. As in Portugal and Spain a quality improvement plan is partly financed through tariffs. If the objective is not met, the company can be penalized. In Sweden they try to achieve a socio-economically acceptable level of continuity and observes the worst performing areas $[1,2]$.

Definition relating to voltage quality. In every country a fraction of worst-served customers exists; today these customers mainly bear costs for poor voltage quality [8].
In only few countries penalties are foreseen in case the voltage limit standards are not met [1]. In others still, the distribution company must take appropriate steps to rectify the causes of the inadequate voltage quality within a given time (in Spain and in Great Britain the period is six months for voltage variations out of prescribed limits).

Table 1 shows the percentage of total consumers in Hungary whose justified voltage complaint (outside the voltage $\pm 7,5 \%$ variation) was not solved within one year.

\begin{tabular}{|l|c|c|}
\hline & 2006 & 2007 \\
\hline \% of total customers & 1,44 & 0,45 \\
\hline
\end{tabular}

Table 1. Justified customers' voltage complaint in Hungary

\section{LATEST RESEARCH TO EXPLORE OF "WORST SERVED" CUSTOMERS}

In the United Kingdom, the regulator, Ofgem voiced its concerns about the poor reliability of supply experienced by a minority of customers and intends to address this issue [9]. Customers located on poor performing circuits may not have benefited from the improvements in overall performance delivered by the incentive scheme. Ofgem appointed market research consultants to undertake a consumer research. As a definition of worst-served customers has not yet been developed, the respondents that took part in this research were recruited on the basis of residing in an area having had and being able to recollect experiencing 15 or more interruptions within a three-year period 10,11].

Ofgem considers that changes to the guaranteed standards of performance would be unlikely to deliver the desired outcome of improvements in performance for worst served customers. As worst served customers represent such a small proportion of the total customer base, the cost of improvement program would outweigh the cost of reasonable penalty payments. Therefore, the customers would continue to receive additional penalty payments but would most likely see little or no improvement in performance [12].

Ofgem considers, but not yet decided, the most appropriate definition of worst served customers is those customers that experience greater than or equal to a given number of interruptions within a year and proposes a threshold of greater than or equal to five interruptions (on average over a three year period) to be most appropriate [12]. It is worth mentioning that in UK one of the GSs relates to supply interruption: four or more separate interruptions each lasting 3 or more hours in any single year. If it is not met a payment must be made: $£ 54$ (£50) for domestic and non- domestic customers. 


\section{CONCLUSONS AND PROPOSALS}

There is no standardized definition of worst-served customers but several approaches exist to define the worst-served customers taking account the national culture and regulatory practice. Most of the approaches relate to the continuity (reliability) of supply and it covers:

- Continuity target both number and duration of long (rarely short) interruptions of individual customer in form of GS.

- Latest research in UK focused on numbers of long interruption and looking for incentives not within the frameworks of GS.

- Another used applied way is to set continuity of supply standards relating to maximum percentage of users subject to a maximum number of interruptions (or minutes lost) in one year.

- Sometimes the definition pertains to worstperforming feeders or area.

Worst-served customers could suffer from not only continuity, but commercial and voltage quality, too. Definitions applied in the industry fails to address all of the three qualities. Therefore the definition of worstserved customers should cover:

- Commercial quality target for the most important services (e.g. answer to customer, time for connection),

- Voltage minimal requirements (e.g. time to supply the prescribed quality).

In connection with guaranteed standards it is worth mentioning and proposing that GS shall really protect consumers if automatic payment is applied. Payment on request is not enough for protecting consumers.

Taking into account that the present economic incentive regulation would outweigh the cost of penalty payments if not meeting GS, the GS would be unlikely to deliver the desired outcome of improvements in performance for worst served customers. Therefore it is worth considering the following:

- GS could be taken as threshold of worse-served customers.

- Maybe it is necessary to set stricter targets than GS that is targets for worst-served customers, e.g. number of interruptions.
- To improve the stricter thresholds regulator can apply incentive or fine if necessary

The number of customers receiving services below the worst-served level thresholds ought to be published in the performance reports.

\section{REFERENCES}

[1] Council of European Energy Regulators, CEER, 2005, Third benchmarking report on quality of electricity supply. Available on the website: www.energy-regulators.eu.

[2] E.Fumagali-L.Lo Schiavo-F.Delestre, 2007, Service Quality Regulation in Electricity Distribution and Retail, 2007, Springer Berlin Heidelberg New York p 5-6

[3] Don Roberts and Xavier Davis, 2003, CustomerOriented Reliability Dec 1, Memphis Light, Gas and Water

[4] UNIPEDE, 1997, "Availability of Supply indices", Unipede Distribution Study Committee 50.05. DISQUAL.

[5] IEEE Power Engineering Society, 2003: Guide for Electric Power Distribution Reliability Indices - IEEE standard 1366-2003.

[6] Edison Electric Institute, 2005, State of Distribution reliability regulation in the United States.

[7] Commonwealth of Kentucky, 2008, An investigation of the reliability measures of Kentucky. Available psc.ky.gov/pscscf/2006\%20cases/200600494/Clark_Report_040108.pdf

[8] Luca Lo Schiavo, 2007, Quality of electricity supply: initiatives of European energy regulators www.cired2007.be/ppt/rt/S6_RT6c-3_CEER.ppt

[9] Ofgem, 2008, Electricity Distribution Price Control Review. Initial consultation document http://www.ofgem.gov.uk/Networks/ElecDist/PriceCn trls/DPCR5/Documents1/Initial\%20consultation\%20d ocument.pdf

[10] James Hope, 2008, DPCR5 Consumer First research: Findings from the qualitative, quantitative and worst served work-strands CEER Meeting, Lissabon, 12 September 2008

[11] Accent: Worst Served Customers, Final Report September 2008 1837rep03 final doc MM 12.09.08

[12] Ofgem, 2008, Electricity distribution price control review. Policy paper, Ref: 159/08 and supplementary appendices 5 www. ofgem.gov.uk 